\title{
Tumor specific hypoxia-activated expression of cardiac peptides using an engineered subtype of Escherichia coli suppresses tumor growth, angiogenesis and metastasis in mouse model of breast cancer
}

Behrad Darvishi ${ }^{1}$, Mitra samadi ${ }^{1}$, Keivan Majidzadeh ${ }^{1}$, malihe salehi ${ }^{1}$, Neda Jalili ${ }^{2}$, Zeinab Noorinejad $^{3}$, Marjan mosayebzadeh ${ }^{1}$, Ahad mohamadnajad ${ }^{4}$, Azade Sharifkhatibi ${ }^{1}$, Shima Moradi $^{1}$, and Leila Farahmand ${ }^{1}$

${ }^{1}$ Breast cancer research center motamed cancer institute

${ }^{2}$ Breast cancer research centaer motamed cancer institute

${ }^{3}$ breast cancer research center motamed cancer institute

${ }^{4}$ Cancer Institute of Iran, Tehran University of Medical Sciences

April 28, 2020

\begin{abstract}
Background Application of genetically modified non-pathogenic bacteria expressing specific anti-tumor proteins under certain conditions specific to tumors is an effective approach for selective targeting of tumors. We developed here, for the first time, a novel spatiotemporal cancer targeted therapy applying engineered E. coli bacteria with capability of expressing cardiac peptides under hypoxic conditions of tumor. Method E. coli BW25133 was transformed with construction of co-expressing cardiac hormones and GFP. Bacteria bearing constructs were then IV administered in mice bearing tumors and then tumor localization, as well as tumor proliferation, invasion and angiogenesis biomarkers (Ki-67, VEGFR, CD31and MMP9), changes in cytokine profile, suppression of tumor growth and survival were analyzed. Results IV Administered bacteria bearing constructs could specifically localize at tumor site and express cardiac peptides under hypoxic conditions. Administration of bacteria significantly enhanced survival rate, suppressed tumor progression and lowered expression levels of MMP-9, VEGFR2, CD31and Ki67 as potent markers for angiogenesis, tumor proliferation and metastasis. Furthermore, applied bacteria resulted in significant reduction in the expression of IL-1 $\beta$, IL-6, GC-SF, IL-12 and TNF- $\alpha$ proinflammatory cytokines, whereas increasing IL-10, IL-17A and INF- $\gamma$ cytokines. Conclusion Overall, administration of E. coli bearing cardiac hormone expression construct could effectively suppress tumor growth, angiogenesis, invasion and metastasis while enhancing survival rate in mice model of breast cancer.
\end{abstract}

Tumor specific hypoxia-activated expression of cardiac peptides using an engineered subtype of Escherichia coli suppresses tumor growth, angiogenesis and metastasis in mouse model of breast cancer

Behrad Darvishi ${ }^{1}$, ,Mitra Samadi $^{1}$, Keivan Majidzadeh-A $^{1}$, Malihe Salehi ${ }^{1}$

,Neda jalilii ${ }^{1}$, Zeinab Noorinejad ${ }^{1}$, Marjan Mosayebzadeh $^{1}$, Ahad muhammadnejad $^{2}$, Azadeh Sharif khatibi $^{1}$, Shima Moradi-Kalbolandi ${ }^{1}$, Leila Farahmand ${ }^{1^{*}}$

1. Recombinant Proteins Department, Breast Cancer Research Center, Motamed Cancer Institute, ACECR, Tehran, Iran. 
2. Cancer Biology Research Center, Cancer Institute of Iran, Tehran University of Medical Sciences, Tehran, Iran.

* Corresponding author: Leila FarahmandEmail: laylafarahmand@gmail.com

Address: No.146, South Gandi Ave, Vanak Sq, Tehran, Iran

Fax: $+9821-88679402$

\section{Abstract}

\section{Background}

Application of genetically modified non-pathogenic bacteria expressing specific anti-tumor proteins under certain conditions specific to tumors is an effective approach for selective targeting of tumors. We developed here, for the first time, a novel spatiotemporal cancer targeted therapy applying engineered E. coli bacteria with capability of expressing cardiac peptides under hypoxic conditions of tumor.

\section{Method}

E. coli BW25133 was transformed with construction of co-expressing cardiac hormones and GFP. Bacteria bearing constructs were then IV administered in mice bearing tumors and then tumor localization, as well as tumor proliferation, invasion and angiogenesis biomarkers (Ki-67, VEGFR, CD31and MMP9), changes in cytokine profile, suppression of tumor growth and survival were analyzed.

\section{Results}

IV Administered bacteria bearing constructs could specifically localize at tumor site and express cardiac peptides under hypoxic conditions. Administration of bacteria significantly enhanced survival rate, suppressed tumor progression and lowered expression levels of MMP-9, VEGFR2, CD31and Ki67 as potent markers for angiogenesis, tumor proliferation and metastasis. Furthermore, applied bacteria resulted in significant reduction in the expression of IL-1 $\beta$, IL-6, GC-SF, IL-12 and TNF- $\alpha$ proinflammatory cytokines, whereas increasing IL-10, IL-17A and INF- $\gamma$ cytokines.

\section{Conclusion}

Overall, administration of E. coli bearing cardiac hormone expression construct could effectively suppress tumor growth, angiogenesis, invasion and metastasis while enhancing survival rate in mice model of breast cancer.

\section{Keywords}

Cardiac peptides, cytokines, MMP9, angiogenesis, VEGFR2, CD31

\section{List of abbreviations}

TNBC: triple negative breast cancer

LANP: Long acting natriuretic peptide

KP: kaliuretic peptide

VD: vessel dilator

SCLC: human small-cell lung carcinomas

E. coli: Escherichia coli

GFP: green fluorescent protein

RBS: ribosomal binding site

pET-CP/GFP: cardiac peptide/GFP expressing plasmid DNA 
LB: Luria-Bertani

MTT: 3-(4,5-dimethylthiazol-2-yl)-2,5-diphenyl tetrazolium bromide

FITC: Fluorescein isothiocyanate

7-AAD: 7-aminoactinomycin D

SMMT; spontaneous mouse mammary tumor

ELISA: Enzyme-Linked ImmunoSorbent Assay

CFU: colony forming units

$\mathrm{H} \& \mathrm{E}$ : hematoxylin and eosin

IHC: Immunohistochemistry

TILs: tumor infiltrating lymphocytes

SDS-PAGE: sodium dodecyl sulfate polyacrylamide gel electrophoresis

IL: Interleukin

TNF- $\alpha$ : Tumor necrosis factor $\alpha$

INF- $\gamma$ : Interferon $\gamma$

MMP9: matrix metalloproteinase 9

VEGFR2: vascular endothelial growth factor receptor 2

ECM: extracellular matrix

CD31: cluster of differentiation 31

\section{Introduction}

An excellent approach for spatiotemporal targeting of tumors, regardless of their origin or location, is application of genetically modified non-pathogenic anaerobic bacteria (e.g. Salmonella, E. coli , Clostridium, Bifidobacterium ) which can be preferentially localized and proliferative in tumor site (1). Contrary to the conventional cancer therapies, bacteria can also penetrate easily in tumor site and be engineered in a way to respond in conditions specific to the tumors (2). Despite this, rendering these genera of bacteria in to non-toxic forms for human results in attenuation of their toxicity against cancer cells (3). Therefore, numerous strategies have been applied for further improving their anti-tumoral activities. One such a strategy is to engineer bacteria to secret specific group of cytolytic proteins such as toxins (e.g. Staphylococcus aureus alpha hemolysin) or enzymes with capability of converting non-toxic prodrugs in to cytotoxic ones (e.g. $E$. coli cytosine deaminase, converting 5-Fluorocytosine in to 5-Fluorouracil) (4).

An even more specific cancer targeted therapy could be achieved through the application of specific promoters capable of controlling gene expression in response to specific inducers such as hypoxia. As a common feature of most aggressive tumors, hypoxia is clinically associated with poor clinical outcomes and development of resistance to most chemotherapeutics and radiotherapy (5). Recently, Nasr and Eidgahi have successfully developed an engineered nirB promoter without any responding region for nitrite and nitrate, making it preferable and selective for expression only under hypoxic tumor condition but not in the presence of chemical inducers (6).

Long acting natriuretic peptide (LANP) together with kaliuretic peptide (KP) and vessel dilator (VDL) comprise three of the four cardiac peptides which have shown to suppress the growth of up to $97 \%$ of cancer cells in vitro. $80 \%$ of human pancreatic adenocarcinomas together with $86 \%$ of human small-cell lung carcinomas (SCLC) have shown to be successfully eradicated in athymic mice through the application of these peptides. Furthermore, administration of cardiac peptides significantly reduced the number of human 
breast adenocarcinoma cells within $24 \mathrm{~h}$ of application in vitro. Among these peptides, Vessel dilator appears to hold the strongest anticancer property, reducing up to $97 \%$ of human prostate cancer cell throughout the first 24 hours (7-9).

In presented study, we developed a novel spatiotemporal cancer targeted therapy applying engineeredE.coli bacteria with capability of expressing cardiac peptides under hypoxic condition of tumor. Creating a hypoxiainducible system, the cardiac peptide genes were coupled to the synthetic nirB promoter sequence reported by Nasr and Eghbali. The stimulation of synthetic nirB promoter was demonstrated using fluorometry and the expression and function of cardiac peptides were demonstrated by immunoblotting and cell culturing. A murine breast cancer model was used to investigate the potency of hypoxia-inducible cardiac peptide secretion using $E$. coli in suppressing tumor growth.

\section{Material and method}

\section{Ethics statement concerning animal work}

All animal experiments were complied with the ARRIVE guidelines and carried out in accordance to the National Institutes of Health guide for the care and use of Laboratory animals (NIH Publications No. 8023, revised 1978). Mice were euthanized by cervical dislocation following completion of tests. the whole study and included procedures were approved by the ethics committee of the Iranian academic center for education, culture and research (ACECR).

\section{Development of plasmid constructs}

A polycistronic cassette, expressing KP, VD and LANP genes in tandem under the control of synthetic nirB promoter reported by Nasr and Eidgahi was designed based on the ANP preprohormone sequence (Genbank accession number: NP_006163). For visualization of bacteria at tumor sites, another polycistronic system expressing green fluorescent protein (GFP) was linked to the previous cassette through a Lambda transcription termination sequence. A ribosomal binding site (RBS) sequence was included upstream of GFP and each of the three gene sequences. For facilitating excretion of expressed cardiac peptides, an immunoglobulin $x$ chain signal peptide (IgK) leader sequence was embedded between RBS and each of the gene's sequence. A terminating codon was also located at the end of each gene sequence. Lambda transcription termination sequence (a rho independent transcription termination sequence) was allocated between the two polycistronic expressing systems to separate expression of corresponding mRNAs from each other. The DNA sequence of GFP was obtained from the GFP commercial plasmid pLOX-EWgfp and fused to the rest of the construct synthesized by Cinnagen Inc. (Iran) performing SOEing-PCR. At the end, the flanking sequences of pET-32 Ek/LIC vector was added to the endings of the construct performing another round of PCR and the construct was cloned into the vector based on previously described method (10).

\section{Transformation of thecardiac peptide/GFP expressing plasmid DNA (pET-CP/GFP)}

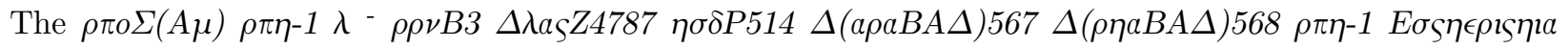
sole strand, BW25133, was grown in Luria-Bertani (LB) media at $37{ }^{\circ} \mathrm{C}$ until mid-log phase, and then harvested at $4 \mathrm{degC}$. After confirmation of correct cloning, pET-CP/GFP constructs were transformed in to E. coli BW25133 strain using calcium chloride method. Bacteria were then maintained and selected in LB media applying $50 \mathrm{mg} / \mathrm{ml}$ Ampicillin and $10 \mathrm{mg} / \mathrm{ml}$ tetracycline. Confirming function of synthetic nirB promoter, bacteria transformed with pET-CP/GFP were grown overnight on LB medium containing 50 $\mathrm{mg} / \mathrm{ml}$ Ampicillin and $10 \mathrm{mg} / \mathrm{ml}$ tetracycline at $37 \mathrm{degC}$. The anaerobic condition was induced by bubbling filtered helium gas through the medium for $10 \mathrm{~min}$. plates were then completely sealed in a way that no air could pass to the medium and incubated for $24 \mathrm{~h}$ at $37 \mathrm{deg}$. fluorescent microscope imaging was used to detect GFP proteins expressed by bacteria.

\section{Toxicity of bacterial secreted cardiac protein on breast cancer cell line}

MCF-7 breast cancer cell line was used for evaluating the anti-proliferative effects of expressed cardiac peptides. Briefly, 96 well microtiter plates were inoculated with 200 
$\mu \mathrm{L}$ growth media containing a density of $2 \times 10^{5}$ cells and then incubated for $24 \mathrm{~h}$ under $5 \% \mathrm{CO} 2$ in humidified air. Bacteria bearing cardiac peptide construct and bacteria without construct were grow on LB agar media under hypoxic condition for $48 \mathrm{~h}$ and then, the bacteria growth media were added to the cells. following $48 \mathrm{~h}$ of exposure, $10 \mu \mathrm{L}$ of 3-(4,5-dimethylthiazol-2-yl)-2,5-diphenyl tetrazolium bromide (MTT) (Sigma Aldrich) with the concentration of $0.05 \mathrm{mg} / \mathrm{mL}$ was added to the media. After $4 \mathrm{~h}$ incubation at $37^{\circ} \mathrm{C}$, whole medium was removed and replaced with $200 \mu \mathrm{L}$ DMSO to dissolve water-insoluble formazan salts. The Absorbance was then read at $570 \mathrm{~nm}$ using a 96 -well plate spectrophotometer.

\section{Annexin V-FITC/propidium iodide flow cytometry}

quantification of apoptosis was carried out by flowcytometry. Briefly MCF-7 cells were treated with $48 \mathrm{~h}$ growth media of bacteria bearing construct and bacteria without construct as well as PBS for $48 \mathrm{~h}$ and then double stained with Annexin V-FITC/ 7-aminoactinomycin D (7-AAD). Fluorescence was then detected with a FACS Calibur flow cytometer (BD Biosciences, USA). Annexin $\mathrm{V}^{+} / 7-\mathrm{AAD}^{-}$cells were determined as early and Annexin $\mathrm{V}^{+} / 7-\mathrm{AAD}^{+}$cells as late apoptotic cells.

\section{In-vivo Breast cancer tumor model}

In-vivo Breast cancer tumor model was developed based on a previously established method by Noori et al. (11). A female Balb/c mouse bearing spontaneous mouse mammary tumor (SMMT) was obtained form Iranian institute of Pasteur. Previous data indicated that SMMT effectively resembles characteristics of Iranian patient's invasive ductal carcinoma (12). SMMT was then carefully separated, dissected into smaller pieces with sizes of less than $0.5 \mathrm{~cm}^{3}$ and subsequently, transplanted to $6-8$ weak old healthy syngeneic Balb/c mice by surgery. 12 days after transplantation, when tumors reached to the size of $500 \mathrm{~mm}^{3}, 10^{7}$ and $10^{8} \mathrm{cfu} / \mathrm{g}$ BW25133 bacteria bearing cardiac hormone expressing construct, as well as $10^{8}$ bacteria without construct and PBS as control were injected systemically to each mouse through the tail vein. A total of three Injections, each with a 4-day interval was performed. To evaluate the effect of treatment on tumor growth delay, tumor volume was measured on predetermined intervals according to the following formula:

$\mathrm{V}\left(\mathrm{mm}^{3}\right)=\mathrm{a}^{2} \times \mathrm{b} \times 0.5$

In which $\mathrm{a}$ and $\mathrm{b}$ represents the shorter and longer diameters $(\mathrm{mm})$ of the tumor respectively. The results were then compared to the PBS and bacteria without construct receiving groups.

Cytokine/chemokine profile measurement by multiplex Enzyme-Linked ImmunoSorbent Assay (ELISA) array

Alterations in expression of major pro- and anti-inflammatory cytokines and chemokines in response to treatment with injection of bacteria was evaluated by multiplex ELISA kits (Cat No. MEM-004A, Qiagen). Briefly, peripheral blood of tumor bearing mice receiving PBS and $10^{8} \mathrm{cfu} / \mathrm{g}$ bacteria bearing construct were collected in endotoxin-free EDTA containing collection tubes (BD Vacutainer Plus, US) immediately after completion of treatment period. Plasma was then collected by centrifugation of blood samples at $2500 \times \mathrm{G}$ for $10 \mathrm{~min}$ and kept at $-80^{\circ} \mathrm{C}$ until further use. For analysis, $50 \mu \mathrm{L}$ of thawed samples was added to each well of their respective row. The ELISA procedure was continued according to the manufacturer's instructions and at the end, absorbance of the 96-well plate was read at $450 \mathrm{~nm}$ and $570 \mathrm{~nm}$ utilizing a plate reader. For correction of autofluoresence, the $570 \mathrm{~nm}$ absorbance was subtracted from final absorbance readings. Expression of each cytokine and chemokine was reported as the fold increase above PBS receiving group levels.

\section{Histological observations and Biodistribution of bacteria in normal and tumor tissues}

Evaluation of Bacterial biodistribution in tumor and normal tissues was carried out $48 \mathrm{~h}$ after tail vain injection of $10^{8} \mathrm{cfu} / \mathrm{g}$ BW25133 bacteria in mice. livers as the highest perfused normal organ and tumors were first excised and fixed in $10 \%$ formalin solution. The specimens were then paraffin embedded, sectioned and stained with gram stain and visualized at $100 \times$ using a light microscope. For counting the number of bacteria in normal and tumor tissues, liver and tumor samples were carefully weighed, homogenized in PBS 
and plated on LB agar. Bacterial concentration was determined by counting the number of CFUs after 24to $48 \mathrm{~h}$ incubation of plates at $37 \mathrm{degC}$. Some of the sections obtained from specimens were stained with hematoxylin and eosin (H\&E) staining solution to evaluate cytotoxic damages induced by cardiac peptide. Presence of BW25133 E. coli in sections was also confirmed by visualization of GFP under fluorescent microscope.

\section{In vivo fluorescence reflectance imaging}

Fluorescence reflectance imaging was utilized to initially evaluate whether bacteria could specifically localize in tumor sites and then to monitor time-course of GFP expression as the indicator of successfull expression of cardiac peptides in tumor site under hypoxic condition. Briefly, mice were IV injected with either PBS (control group) or E. coli BW25133 strain bearing construct with concentration of $10^{8} \mathrm{cfu} / \mathrm{g}$ and images were taken on days $0,1,3$ and 6 , post injection using the FlouVison fluorescence planar imaging system in vivo (Tajhizafarinan Noori Parseh Co., Tehran, Iran). Images from whole body were taken by placing anesthetized mice in the supine position, in the center of the imaging cassette, inside the scanning field of the system. After proper positioning of animals, the imaging cassette was set to the appropriate depth to finely confine anesthetized mice. Finally, the animal body was subjected to the laser beam and emitted non-uniform fluorescent beams were recorded using a highly sensitive thermoelectrically cooled CCD camera located on the same side of the imaged animal.

\section{IHC and histological examination}

At the end of the 24-day treatment period, mice receiving PBS and E. coli BW25133 strain bearing construct with concentration of $10^{8} \mathrm{cfu} / \mathrm{g}$ were sacrificed and tumors equivalent in size from both groups were selected for performing immunohistological analyses. Collected tumors were cut in to $7 \mu \mathrm{m}$ thick section on a microtome and stained using anti-Ki67 monoclonal antibody (ab15580, Abcam, USA), a marker for tumor proliferation; anti-MMP9 monoclonal antibody (ab38898, Abcam, USA), an enzyme involved in inducing angiogenesis and metastasis; anti-VEGF receptor 2 monoclonal antibody (ab2349, Abcam, USA), a proangiogenic growth factor; anti-CD31/PECAM-1 monoclonal antibody (ab24590, Abcam, USA), recognizing platelet-endothelial cell adhesion molecule-1 (PECAM-1) expressed on surface of endothelial cells; anti-CD8 monoclonal antibody (ab209775, Abcam, USA), an specific marker for cytotoxic T-cells; anti-CD4 monoclonal antibody (ab221775, Abcam, USA), an specific marker for helper T-cells; and their specific horseradish peroxidase-conjugated secondary antibody. Antigen recovery was performed in citrate buffer $\mathrm{pH} 6.0$ and then blockade of endogenous peroxidase, as well as non-specific proteins was performed by 40 min incubation with $3 \%$ of $\mathrm{H}_{2} \mathrm{O}_{2}$ and another 40 min with $3 \%$ FBS. At the end, tumor sections were incubated with mentioned antibodies and extend of antigen expression was evaluated using horseradish peroxidase (HRP)-conjugated streptavidin and 2-Solution DAB kit (Life Technologies) according to the companies' manual instructions. Finally, each antigen's expression level was quantified using ImageJ software (NIH, Bethesda, MD, USA). Results were expressed as the mean of at least 5 tumor sections for each antigen.

H\&E staining was also performed for evaluating tumor infiltrating lymphocytes (TILs) according to the previously established protocols. In Brief, initially, percentage of stromal lymphocytes were determined by two separate observers and then stromal TILs were measured as the percentage of immune cells with mononuclear immunological infiltrate characteristics. Findings were classified based on three cut-off points for TIL proportions including 10\%, 30\% and 50\%.

\section{Statistical analysis}

Data are represented as mean \pm their standard deviation. Hypothesis testing was carried out applying Student's $t$-test and results considered significant in the case of $P<0.05$. Survival analysis was done by drawing Kaplan-Meier curves and comparing groups with log-rank test. Hazard ratios were calculated by performing Cox's proportional hazards analysis.

\section{Results}

Design of construct and expression of cardiac peptides and GFP 
The overall structure of the construct co-expressing the cardiac peptides and GFP under the control of synthetic nirB promoter has been represented in figure 1A. Presence of a single band at 550 bp in electrophoresis confirmed correct performance of SOEing PCR (figure 1B). results of colony PCR also confirmed the presence of constructs in recombinant bacteria (figure 1C). following induction of anaerobic condition, collected supernatant of bacteria was concentrated and subjected to SDS-PAGE analysis to evaluate expression of cardiac peptides. As demonstrated in figure $2 \mathrm{~A}$, presence of a single band at about $5 \mathrm{kDa}$ confirmed expression of cardiac peptides. As all the expressed cardiac peptides possess a molecular weight very close to each other, three single bands could have been overlapped together and it is not surprising to observe only one band in SDS-PAGE analysis. Extracted proteins form cytoplasm of bacterial pellets in lysis buffer were also subjected to SDS-PAGE analysis for evaluation of expression of GFP. The band at $27 \mathrm{kDa}$ relates to the molecular weight of GFP which is absent in the bacterial samples non-transformed with the cassette (figure 2B).

\section{Anti-proliferative and pro-apoptotic effects of bacterial secreted cardiac protein}

Incubation of MCF-7 human mammary carcinoma cells with growth media of bacteria bearing construct under hypoxic condition for a 48-h period, promoted both cell death and apoptosis. The results of MTT assay demonstrated that administration of the growth media of bacteria bearing construct, resulted in inhibition of growth in about $40 \%$ of MCF-7 cells which was significantly higher compared to growth media of bacteria without construct and PBS (25\% and 5\% inhibition respectively). (fig. 3A). The Annexin-V/7-AAD flow cytometry analysis also demonstrated that treating MCF-7 cells with the growth media of bacteria bearing construct significantly increases rate of apoptosis compare to bacteria without construct and PBS treated group (Fig. 3B).

\section{Preferential colonization of E. coli BW25113 within tumor}

Systemic administration of E. coli BW25113 in syngeneic mice bearing spontaneous mammary tumors resulted in elicit and preferential colonization of bacteria at tumor site. As depicted in figure $4 \mathrm{~A}$, bacteria accumulated in tumor site with an approximately 15 -fold greater density compare to the liver. Furthermore, microscopic examination of tissue slides also confirmed that E. coli was present in all analyzed tumors. Accumulation of E. coli BW25113 was more significant at necrotic sites of tumor.

Confirming hypoxia-activated expression of cardiac peptides following tumor specific localization of E. coli Bw25113 using In vivo fluorescence reflectance imaging

E. coli BW25113 bearing cardiac hormone expressing constructs and PBS were injected to the mice and images were collected immediately and $24 \mathrm{~h}, 3$ days and 6 days post injection. As depicted in figure 4B, no significant differences in emitted fluorescent intensity was observed between two group during the first $24 \mathrm{~h}$ post injection. On day 3 however, emitted fluorescent from bacteria receiving group slightly grew and become a bit higher than those observed in PBS receiving group. Continuing similar trend, on day 6 post injection, emitted fluorescent from bacteria receiving group reached to its maximum and was completely restricted to the tumor site. Regarding other organelles, no significant changes in fluorescent intensity was observed during image acquisition period neither in control nor in bacteria receiving groups. These observations further confirm site specific colonization of bacteria and expression of GFP as an indicator of successful expression of construct in hypoxic condition.

\section{Tumor growth retardation potential ofbacteria expressing cardiac protein}

The delay in tumor growth was evaluated over a 24-day period after the administration of bacteria bearing construct with concentrations equal to $10^{7}$ and $10^{8} \mathrm{cfu} / \mathrm{g}$, bacteria without construct in concentration equal to $10^{8} \mathrm{cfu} / \mathrm{g}$ and PBS as the control group. Based on results, administration of bacteria without construct didn't result in any significant tumor growth retardation compared to PBS receiving group. Therefore, bacteria per se were not capable of suppressing tumor growth speed. Contrarily, administration of bacteria bearing constructs, even at concentration equal to $10^{7} \mathrm{cfu} / \mathrm{g}$ resulted in a significantly greater delay in tumor growth compare to PBS group. Furthermore, increasing the concentration of bacteria from $10^{7} \mathrm{cfu} / \mathrm{g}$ to $10^{8} \mathrm{cfu} / \mathrm{g}$ 
resulted in more enhanced tumor suppression. As depicted in figure 5A, differences in delaying tumor growth between two concentrations of bacteria bearing construct become significant on day 15 from beginning of treatment. Furthermore, the differences between delaying in tumor growth in group receiving concentrations of bacteria equal to $10^{8} \mathrm{cfu} / \mathrm{g}$ and PBS group become significant on day 12 . However, similar significancy for the group receiving bacteria bearing construct at $10^{7} \mathrm{cfu} / \mathrm{g}$ took place on day 15 . Based on these observations we concluded that first, the delay in tumor growth observed with administration of bacteria bearing construct is mainly due to the secretion of cardiac proteins. Second, increasing concentration of administered bacteria may result in enhancement of production and secretion of cardiac peptides. Administration of bacteria bearing constructs at both concentrations also significantly enhanced survival rate as observed in KaplanMeyer analysis (figure 5B).

\section{Alteration in pro-inflammatory cytokine profile}

We also examined the effect of bacterial treatment on expression of pro-inflammatory cytokines. As depicted in figure 6, administration of bacteria bearing construct at concentrations equal to $10^{8} \mathrm{cfu} / \mathrm{g}$ to the mice bearing spontaneous breast tumors, resulted in a significant decline in expression of pro-inflammatory cytokines, including IL- $1 \alpha$, IL-1 $\beta$, IL-6, IL-12, TNF- $\alpha$, G-CSF and GM-CSF while increasing expression of IL-17A, IL-10 and INF- $\gamma$ compared to the PBS receiving group. No significant changes were observed in expression profile of IL-2 pro-inflammatory cytokine. Therefore, treatment with bacteria bearing cardiac hormone expressing construct could increase expression of some of the most important cytokines of both $\mathrm{T}_{\mathrm{h}} 1$ and $\mathrm{T}_{\mathrm{h}} 2$ cells (INF- $\gamma$ and IL-10 respectively).

\section{Immunohistochemistry, quantification of micro-vessel density and $\mathbf{H} \& \mathbf{E}$ staining results}

As illustrated in Figure 7, MMP9 expression was significantly decreased in mice receiving bacteria bearing construct at concentrations equal to $10^{8} \mathrm{cfu} / \mathrm{g}$ compared to PBS receiving group. Furthermore, expression of VEGF receptor 2 (VEGFR2) and Ki-67 were also significantly decreased in response to therapy. Also, comparing state of CD31 expression as a marker of micro-vessels density demonstrated a significant decline in mice receiving bacteria bearing construct at concentrations equal to $10^{8} \mathrm{cfu} / \mathrm{g}$ compare to control group. Finally, the number of TILs and CD8+ TILs were significantly increased in mice treated with bacteria bearing construct at concentration equal to $10^{8} \mathrm{cfu} / \mathrm{g}$.

\section{Discussion}

Based on observed results, it is elicit that E. coli BW25113 is capable of expressing and secreting cardiac peptides including LANP, VD and KP as well as GFP as an indicator protein under the control of hypoxic or anaerobic condition. Secreted cardiac peptides by interacting with their receptors on carcinoma cells result in induction of apoptosis and cell death. The main advantage of presented approach is selectivity in both targeting tumor site and inducing cytotoxicity only in hypoxic areas of tumor. As demonstrated in sections collected from tumor tissue and liver, bacteria preferentially localized in tumor site with a significantly greater density. Furthermore, activation of protein expression only at hypoxic condition, prohibits expression of cardiac proteins and observation of undesired side effects at normally perfused body organs including liver, lung, heart, etc. Other studies, including the one performed by Stritzker et al., have also demonstrated that different strains of e. coli are capable of preferentially colonizing in tumor site (13).

Based on the study performed by Kim et al, contrary to the salmonella typhimurium which is frequently applied in bacterial tumor targeting, E. coli strain K-12 (MG1655) is not capable of suppressing tumor growth per se (14). Our results also clearly demonstrated that administration of e. coli BW25113 even at high doses didn't significantly suppressed tumor growth. As the only difference between bacteria bearing construct and untransformed ones is the capability of expressing cardiac proteins, differences in tumor growth delay may be attributed to the expressed cardiac peptides under hypoxic condition of tumor.

Several in vitro studies have clearly indicated the cytotoxic effects of cardiac peptides against malignant cells. Based on studies performed by Vesely et al, four cardiac peptides including LANP, KP, VD and ANP demonstrate significant anti-proliferative effects against breast, prostate, lung, brain and renal carcinomas 
(15). Reported by Sun et al., VD and KP demonstrate their anti-cancer activities in prostate cancer through inhibiting RAS activity (16). Furthermore, LANP is capable of inhibiting $80-90 \%$ of MEK1/2 and ERK1/2 activity in prostate cancer cells $(17,18)$. As ERK plays an important role in controlling apoptosis, inhibition of its phosphorylation and activation by LANP may partly explain its pro-apoptotic mechanism of action (19).

Currently, multiple studies have addressed the role of cytokines in cancer proliferation, invasion and metastasis. For instance, it has been shown that high circulating levels of IL- $1 \beta$ and IL- 6 are unfavorable prognostic indicators and directly interrelate with higher rate of recurrence and clinical stage in patients with breast cancer (20-22). Contrarily, overexpression of INF- $\gamma$ has shown to promote cell death through up-regulating caspases and demonstrate significant antiangiogenic activity (23-25). Administration of bacteria bearing construct resulted in a complex effect on expression of pro-inflammatory cytokines. Many studies have shown that cardiac peptides demonstrate an anti-inflammatory behavior. For instance, it has been shown that ANP could down regulate expression of TNF- $\alpha$ which has an important role in alloreactivity of T-cells $(26$, 27). Furthermore, ANP can also enhance expression of IL-10 which in turn, may result in downregulation of IL-2 and proliferation of T-cells $(28,29)$. IL- 6 concentration has also been shown to decline in response to administration of ANP (29). Finally, administration of ANP together with LPS decreases production of IL-12 by dendritic cells and generates fewer INF- $\gamma$ positive T cells and much more IL-4 positive ones (30). Consistent with these reports, here we also observed a significant decline in peripheral blood concentrations of pro-inflammatory cytokines including IL-1a, IL-1b, IL-6, IL-12 and TNF-a. contrarily the concentrations of IL-10 and INF- $\gamma$ were significantly increased in response to therapy.

Angiogenesis is a firmly controlled process, crucial for tumor expansion beyond 2 to $3 \mathrm{~mm}$ in diameter and a critical step of tumor invasion and metastasis (31). Different studies have now suggested a critical role for MMP9 in formation of a proper microenvironment for promotion of tumor growth and angiogenesis through enhancing the association between VEGF and VEGFR2 $(32,33)$. This mainly takes place through the proteolytic cleavage and subsequent release of extracellular matrix (ECM) bound VEGF by MMP9 activity which is an important step of angiogenic switch. Based on Bergers et al. inhibitors of MMP9 effectively suppressed tumor growth and reduced angiogenic switching (34). It has also been shown that MMP-cleaved VEGF plays an important role in tumors vascular patterning $(7,35)$. Based on previous reports, cardiac peptides effectively reduce expression of VEGF in vitro (7). In present study, we demonstrated that administration of bacteria expressing cardiac peptides under hypoxic condition could reduce MMP-9 expression in vivo. In parallel, VEGFR2-triggered angiogenesis is considered as a hallmark of cancer progression and metastasis $(36,37)$. Consistent with previous studies, we also found that expression of VEGFR2 was significantly reduced in tumors from mice receiving bacteria bearing cardiac peptides expressing construct (7).

Results from different reports show that high tumor micro-vessel density measured by immunohistochemical analysis of CD31 expression, is correlated with higher rates of lymph node metastasis and poor prognosis (3840). In addition, MMP-9 has widely been mentioned as an important driver of cancer's malignant progression, invasion and metastasis (41). Also, determination of proportion of cancer cells undergoing proliferation by immunohistochemical analysis of Ki-67, has shown that high Ki-67 expression is associated with increased risk of metastasis development as well as poor overall survival (42). As administration of bacteria expressing cardiac peptides could effectively reduce expression of Ki-67, CD31 and MMP-9, it may be an effective therapeutic for preventing from invasion and future development of metastasis. Finally, presence of TILs (especially CD8+ TILs), which demonstrate effective anti-tumoral activity in tumor sites, has shown to be together with a favorable prognosis value in different neoplasms (43-46). Therefore, significant increase in survival rate as observed with Kaplan-Meyer analysis may be partly attributed to the higher number of CD8+ TILs in tumor site, as well as lower expression values of above-mentioned markers.

\section{Conclusion}

Overall, in present study, we demonstrated that IV administration of E. coli BW25113 strain bearing cardiac hormone expression construct could result in tumor specific localization and subsequent hypoxia derived expression of cardiac peptides. This resulted in suppression of tumor growth, significant increase in survival 
rate and decreased expression of MMP-9, VEGFR2, Ki-67 and CD31, all of which propose an antiangiogenic and anti-metastatic activity for the proposed therapy.

\section{Disclosure statement}

The authors declare that there exist no conflict of interests.

\section{References}

1. Pawelek JM, Low KB, Bermudes D. Bacteria as tumour-targeting vectors. The lancet oncology. 2003;4(9):54856.

2. Forbes NS. Engineering the perfect (bacterial) cancer therapy. Nature Reviews Cancer. 2010;10(11):785.

3. Nemunaitis J, Cunningham C, Senzer N, Kuhn J, Cramm J, Litz C, et al. Pilot trial of genetically modified, attenuated Salmonella expressing the E. coli cytosine deaminase gene in refractory cancer patients. Cancer gene therapy. 2003;10(10):737.

4. Jean ATS, Swofford CA, Panteli JT, Brentzel ZJ, Forbes NS. Bacterial delivery of Staphylococcus aureus $\alpha$-hemolysin causes regression and necrosis in murine tumors. Molecular Therapy. 2014;22(7):1266-74.

5. Vaupel P, Mayer A. Hypoxia in cancer: significance and impact on clinical outcome. Cancer and Metastasis Reviews. 2007;26(2):225-39.

6. Nasr R, Eidgahi MRA. Construction of a synthetically engineered nirB promoter for expression of recombinant protein in Escherichia coli. Jundishapur journal of microbiology. 2014;7(7).

7. Nguyen JP, Frost CD, Lane ML, Skelton IV WP, Skelton M, Vesely DL. Novel dual inhibitors of vascular endothelial growth factor and VEGFR2 receptor. European journal of clinical investigation. 2012;42(10):10617 .

8. Vesely D, Clark L, Garces A, McAfee Q, Soto J, Gower Jr W. Novel therapeutic approach for cancer using four cardiovascular hormones. European journal of clinical investigation. 2004;34(10):674-82.

9. Vesely DL. Which of the cardiac natriuretic peptides is most effective for the treatment of congestive heart failure, renal failure and cancer? Clinical and experimental pharmacology and physiology. 2006;33(3):169-76.

10. Farahmand L, Majidzadeh-A K, Sepehrizadeh Z, Mofid MR, Esmaeili R, Yazdi MT. Ligation independent cloning of polycistronic, genetically modified, HuMAb4D5-8 F (ab') 2, in bacterial plasmid. Avicenna journal of medical biotechnology. 2012;4(1):15.

11. Noori S, Hassan ZM. Dihydroartemisinin shift the immune response towards Th1, inhibit the tumor growth in vitro and in vivo. Cellular immunology. 2011;271(1):67-72.

12. Langroudi L, Hassan ZM, Ebtekar M, Mahdavi M, Pakravan N, Noori S. A comparison of low-dose cyclophosphamide treatment with artemisinin treatment in reducing the number of regulatory $\mathrm{T}$ cells in murine breast cancer model. International immunopharmacology. 2010;10(9):1055-61.

13. Stritzker J, Weibel S, Hill PJ, Oelschlaeger TA, Goebel W, Szalay AA. Tumor-specific colonization, tissue distribution, and gene induction by probiotic Escherichia coli Nissle 1917 in live mice. International journal of medical microbiology. 2007;297(3):151-62.

14. Kim J-E, Phan TX, Nguyen VH, Dinh-Vu H-V, Zheng JH, Yun M, et al. Salmonella typhimurium suppresses tumor growth via the pro-inflammatory cytokine interleukin-1 $\beta$. Theranostics. 2015;5(12):1328.

15. Vesely DL. Cardiac hormones for the treatment of cancer. Endocr Relat Cancer. 2013;20:R113-R25.

16. Sun Y, Eichelbaum EJ, Lenz A, Wang H, Vesely DL. Epidermal growth factor's activation of Ras is inhibited by four cardiac hormones. European journal of clinical investigation. 2010;40(5):408-13. 
17. Sun Y, Eichelbaum EJ, Wang H, Vesely DL. Atrial natriuretic peptide and long acting natriuretic peptide inhibit ERK 1/2 in prostate cancer cells. Anticancer research. 2006;26(6B):4143-8.

18. Sun Y, Eichelbaum EJ, Wang H, Vesely DL. Atrial natriuretic peptide and long acting natriuretic peptide inhibit MEK 1/2 activation in human prostate cancer cells. Anticancer research. 2007;27(6B):3813-8.

19. Vesely B, Alli A, Song S, Gower Jr W, Sanchez-Ramos J, Vesely D. Four peptide hormones' specific decrease (up to $97 \%$ ) of human prostate carcinoma cells. European journal of clinical investigation. 2005;35(11):700-10.

20. Kovacs E. Investigation of interleukin-6 (IL-6), soluble IL-6 receptor (sIL-6R) and soluble gp130 (sgp130) in sera of cancer patients. Biomedicine \& pharmacotherapy. 2001;55(7):391-6.

21. Mettler L, Salmassi A, Heyer M, Schmutzier A, Schollmeyer T, Jonat W. Perioperative levels of interleukin-1beta and interleukin-6 in women with breast cancer. Clinical and experimental obstetrics \& gynecology. 2004;31(1):20-2.

22. Kozłowski L, Zakrzewska I, Tokajuk P, Wojtukiewicz M. Concentration of interleukin-6 (IL-6), interleukin8 (IL-8) and interleukin-10 (IL-10) in blood serum of breast cancer patients. Roczniki Akademii Medycznej w Bialymstoku (1995). 2003;48:82-4.

23. Connett JM, Badri L, Giordano TJ, Connett WC, Doherty GM. Interferon regulatory factor 1 (IRF1) and IRF-2 expression in breast cancer tissue microarrays. Journal of interferon \& cytokine research. $2005 ; 25(10): 587-94$.

24. Bouker KB, Skaar TC, Riggins RB, Harburger DS, Fernandez DR, Zwart A, et al. Interferon regulatory factor-1 (IRF-1) exhibits tumor suppressor activities in breast cancer associated with caspase activation and induction of apoptosis. Carcinogenesis. 2005;26(9):1527-35.

25. Sidky YA, Borden EC. Inhibition of angiogenesis by interferons: effects on tumor-and lymphocyteinduced vascular responses. Cancer research. 1987;47(19):5155-61.

26. Kiemer AK, Hartung T, Vollmar AM. cGMP-mediated inhibition of TNF- $\alpha$ production by the atrial natriuretic peptide in murine macrophages. The Journal of Immunology. 2000;165(1):175-81.

27. Ladetzki-Baehs K, Keller M, Kiemer AK, Koch E, Zahler S, Wendel A, et al. Atrial natriuretic peptide, a regulator of nuclear factor- $\varkappa$ B activation in vivo. Endocrinology. 2007;148(1):332-6.

28. Leitman DC, Andresen JW, Catalano RM, Waldman SA, Tuan JJ, Murad F. Atrial natriuretic peptide binding, cross-linking, and stimulation of cyclic GMP accumulation and particulate guanylate cyclase activity in cultured cells. Journal of Biological Chemistry. 1988;263(8):3720-8.

29. Moro C, Klimcáková E, Lolmède K, Berlan M, Lafontan M, Stich V, et al. Atrial natriuretic peptide inhibits the production of adipokines and cytokines linked to inflammation and insulin resistance in human subcutaneous adipose tissue. Diabetologia. 2007;50(5):1038-47.

30. Mohapatra SS, Lockey RF, Vesely DL, Gower Jr WR. Natriuretic peptides and genesis of asthma: an emerging paradigm? Journal of allergy and clinical immunology. 2004;114(3):520-6.

31. Carmeliet P, Jain RK. Angiogenesis in cancer and other diseases. nature. 2000;407(6801):249.

32. Rodríguez-Manzaneque JC, Lane TF, Ortega MA, Hynes RO, Lawler J, Iruela-Arispe ML. Thrombospondin1 suppresses spontaneous tumor growth and inhibits activation of matrix metalloproteinase- 9 and mobilization of vascular endothelial growth factor. Proceedings of the National Academy of Sciences. 2001;98(22):1248590.

33. Mira E, Lacalle RA, Buesa JM, de Buitrago GG, Jiménez-Baranda S, Gómez-Moutón C, et al. Secreted MMP9 promotes angiogenesis more efficiently than constitutive active MMP9 bound to the tumor cell surface. J Cell Sci. 2004;117(9):1847-57. 
34. Bergers G, Brekken R, McMahon G, Vu TH, Itoh T, Tamaki K, et al. Matrix metalloproteinase-9 triggers the angiogenic switch during carcinogenesis. Nature cell biology. 2000;2(10):737.

35. Lee S, Jilani SM, Nikolova GV, Carpizo D, Iruela-Arispe ML. Processing of VEGF-A by matrix metalloproteinases regulates bioavailability and vascular patterning in tumors. The Journal of cell biology. 2005;169(4):681-91.

36. Olsson A-K, Dimberg A, Kreuger J, Claesson-Welsh L. VEGF receptor signalling? In control of vascular function. Nature reviews Molecular cell biology. 2006;7(5):359.

37. Herbert SP, Stainier DY. Molecular control of endothelial cell behaviour during blood vessel morphogenesis. Nature reviews Molecular cell biology. 2011;12(9):551.

38. Weidner N, Semple JP, Welch WR, Folkman J. Tumor angiogenesis and metastasis - correlation in invasive breast carcinoma. New England Journal of Medicine. 1991;324(1):1-8.

39. Hansen S, Grabau DA, Sørensen FB, Bak M, Vach W, Rose C. The prognostic value of angiogenesis by Chalkley counting in a confirmatory study design on 836 breast cancer patients. Clinical cancer research. 2000;6(1):139-46.

40. Jacquemier JD, Penault-Llorca FM, Bertucci F, Sun ZZ, Houvenaeghel GF, Geneix JA, et al. Angiogenesis as a prognostic marker in breast carcinoma with conventional adjuvant chemotherapy: a multiparametric and immunohistochemical analysis. The Journal of Pathology: A Journal of the Pathological Society of Great Britain and Ireland. 1998;184(2):130-5.

41. Huang H. Matrix metalloproteinase-9 (MMP-9) as a cancer biomarker and MMP-9 biosensors: Recent advances. Sensors. 2018;18(10):3249.

42. Green WJ, Ball G, Hulman G, Johnson C, Van Schalwyk G, Ratan HL, et al. KI67 and DLX2 predict increased risk of metastasis formation in prostate cancer-a targeted molecular approach. British journal of cancer. 2016;115(2):236.

43. Mahmoud SM, Paish EC, Powe DG, Macmillan RD, Grainge MJ, Lee AH, et al. Tumor-infiltrating CD8+ lymphocytes predict clinical outcome in breast cancer. Journal of clinical oncology. 2011;29(15):194955.

44. Wang W, Kryczek I, Dostal L, Lin H, Tan L, Zhao L, et al. Effector T cells abrogate stroma-mediated chemoresistance in ovarian cancer. Cell. 2016;165(5):1092-105.

45. Laghi L, Bianchi P, Miranda E, Balladore E, Pacetti V, Grizzi F, et al. CD3+ cells at the invasive margin of deeply invading (pT3-T4) colorectal cancer and risk of post-surgical metastasis: a longitudinal study. The lancet oncology. 2009;10(9):877-84.

46. Galon J, Costes A, Sanchez-Cabo F, Kirilovsky A, Mlecnik B, Lagorce-Pages C, et al. Type, density, and location of immune cells within human colorectal tumors predict clinical outcome. Science. 2006;313(5795):1960-4.

\section{Figure Legends}

Figure 1- (A) The overall structure of the construct co-expressing the cardiac peptides and GFP under the control of synthetic nirB promoter. (B) results of GFP and Cardiac peptide PCR. Lane 1: the DNA size marker, Lane 2: result of GFP PCR, Lane 3: result of cardiac peptides PCR, Lane 4: Negative control (C) Result of SOEing PCR. Presence of a single band at $550 \mathrm{bp}$ in electrophoresis confirmed correct performance of SOEing PCR. Lane 1: the DNA size marker, Lane 2: result of SOEing PCR Lane 3: Negative control(D) Results of colony PCR, confirming the presence of constructs in recombinant bacteria. Lane 1: The negative control (prepared PCR mixture applying water instead of DNA template), Lane 2: the DNA size marker, Lane 3: the positive colonies comprising the cardiac hormone expressing construct. 
Figure 2- Results of SDS-PAGE analysis. (A) Presence of a single band at about $5 \mathrm{kDa}$ confirmed expression of cardiac peptides. Lane 1: protein marker, Lane 2: before induction of hypoxic condition, Lane 3: $24 \mathrm{~h}$ after induction of hypoxic condition.(B) The single band at $27 \mathrm{kDa}$ relates to the molecular weight of GFP which is absent in the bacterial samples non-transformed with the cassette. Lane 1: before induction of hypoxic condition, Lane 2: protein marker, Lane 3: $24 \mathrm{~h}$ after induction of hypoxic condition.

Figure 3- (A) In vitro cytotoxicity of purified cardiac peptides from $48 \mathrm{~h}$ growing media of bacteria bearing construct under hypoxic condition. (B) The results of Annexin-V/7-AAD flow cytometry analysis. Treating MCF-7 cells with cardiac peptides mixture significantly increased rate of apoptosis compare to PBS receiving group (Left: PBS, Middle: media of bacteria without construct, Right: cardiac peptides purified from media of bacteria bearing construct).

Figure 4- (A) Bacterial concentration in tumor tissue and liver of mice $48 \mathrm{~h}$ after IV administration of $10^{8}$ $\mathrm{cfu} / \mathrm{g}$ bacteria. Accumulation in tumor site was about 15-fold greater compare to the liver. (B) Results of In vivo fluorescence reflectance imaging $0,1,3$ and 6 days post injection. on day 6 post injection, Maximum New fluorescent emissions were completely restricted to the tumor site and reached to its maximum on day 6 post injection.

Figure 5- (A) suppression of tumor growth by administration of different concentrations of bacteria bearing construct. Increase in concentration of administered bacteria significantly enhanced tumor suppression potency. Ns: not significant, ****: $\mathrm{P}<0.0001$ compared to $\mathrm{PBS}$, \#\#\#\#: $\mathrm{P}<0.0001$ compared to bacteria bearing construct at concentrations equal to $10^{7} \mathrm{cfu} / \mathrm{g}(\mathbf{B})$ Results of Kaplan-Meyer analysis demonstrating a higher rate of survival in mice receiving bacteria bearing construct compared to PBS and bacteria without construct.

Figure 6- Alteration in expression of pro-inflammatory cytokines in response to bacterial therapy. Results are expressed as fold changes compared to control group receiving PBS. *: $\mathrm{P}<0.05,{ }^{* *}: \mathrm{P}<0.01,{ }^{* * *}: \mathrm{P}<0.001$ compared to control group.

Figure 7- Results of immunohistochemical analysis, quantification of micro-vessel density and H\&E staining.

(A)

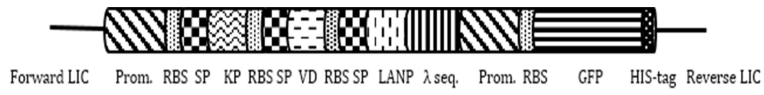

(B)

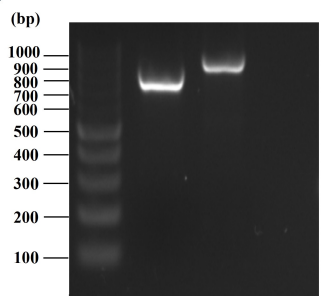

(C)

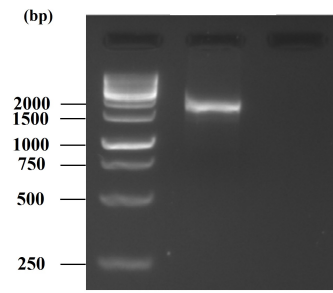

(D)

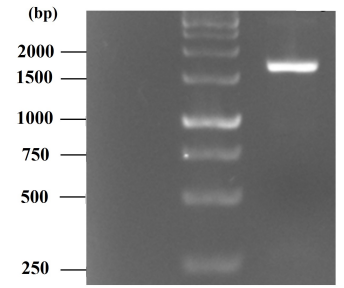



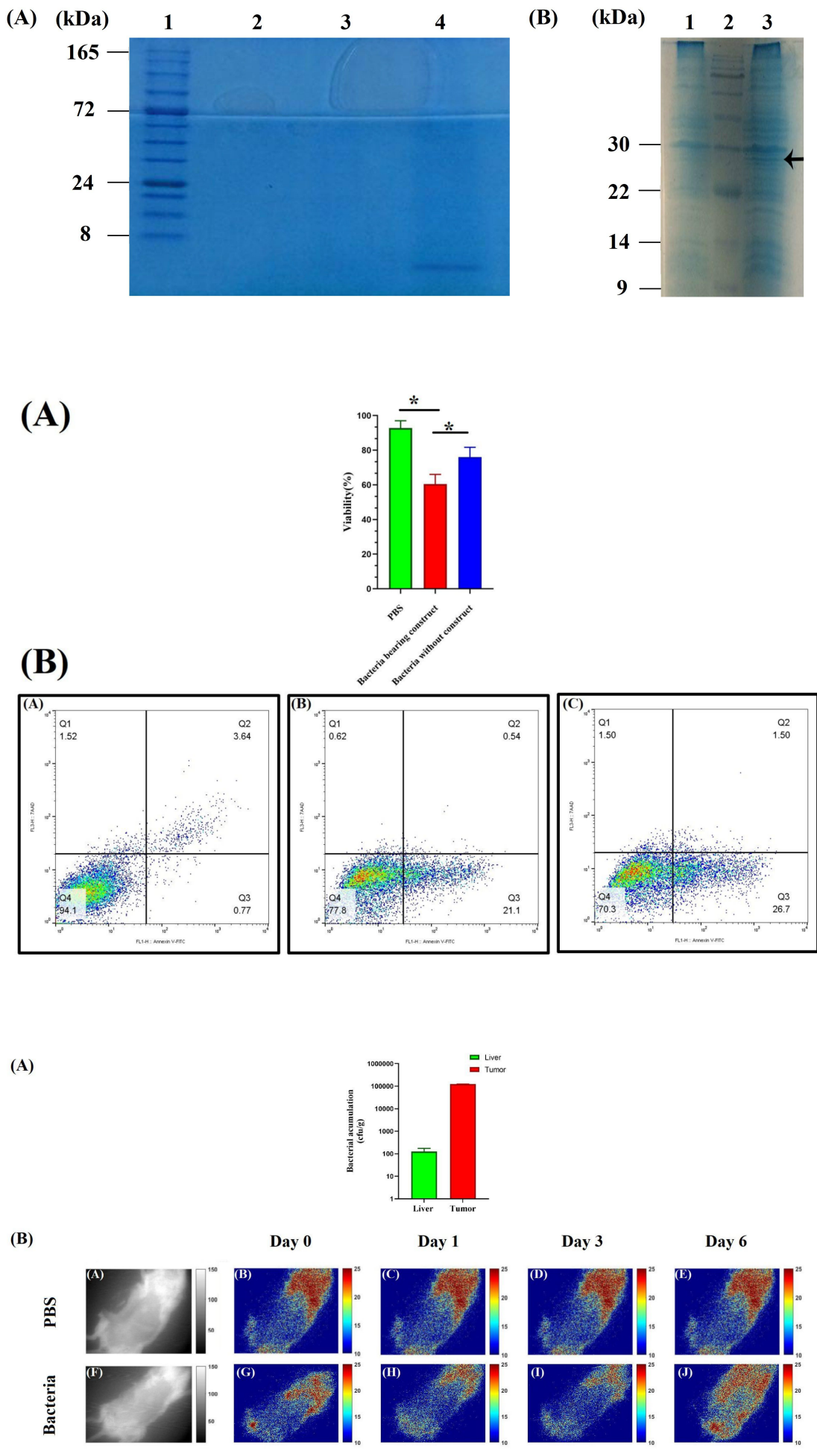

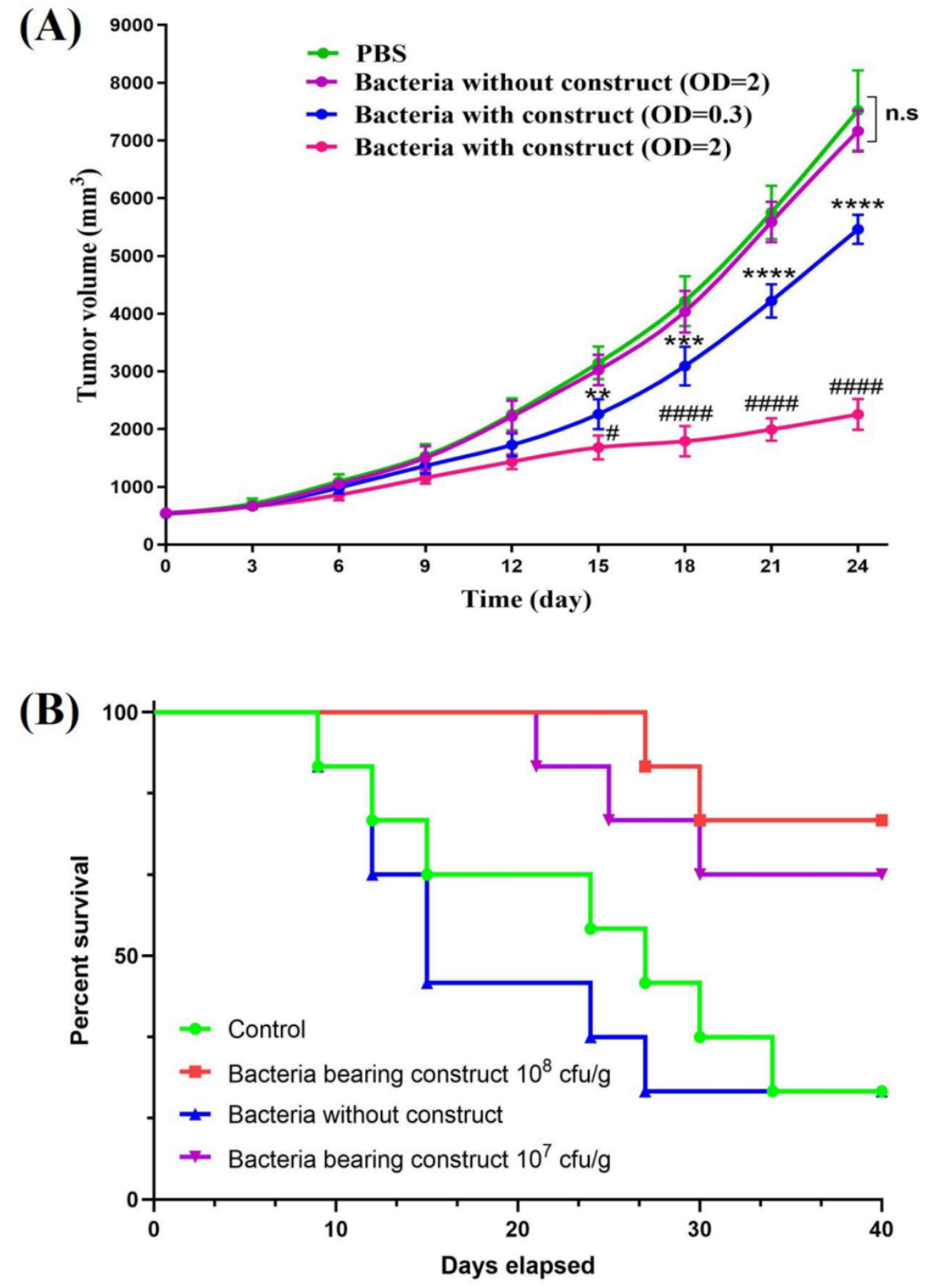

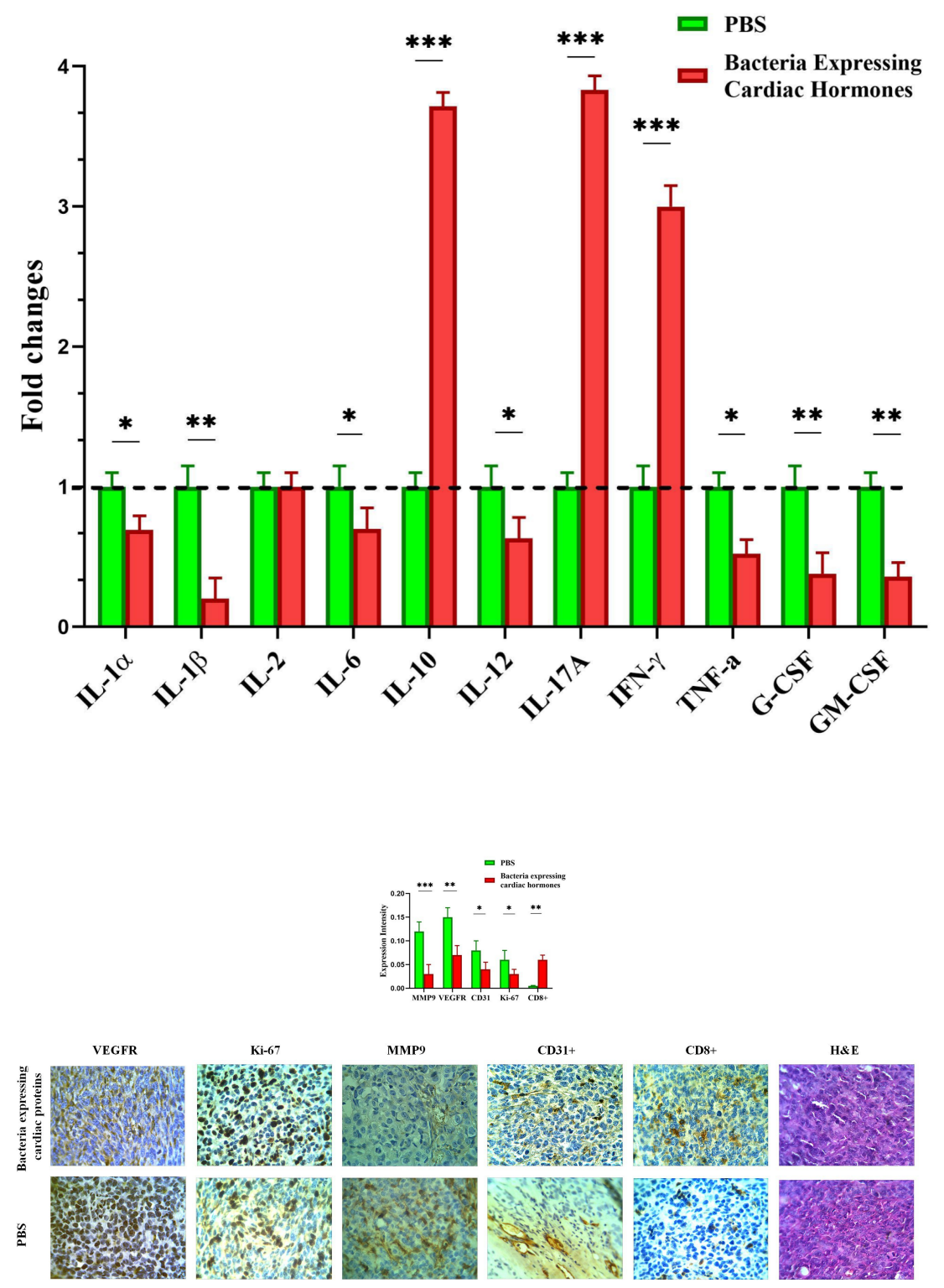